\title{
Disorder in the Representational Warehouse
}

\author{
Victoria McGeer \\ Princeton University
}

\author{
Eric Schwitzgebel \\ University of California at Riverside
}

\begin{abstract}
Although developmental psychologists are generally happy to endorse dissociationist and gradualist views of development like Woolley's (2006), the design and interpretation of developmental research often suggests an implicit commitment to a cleaner, less dissociative, sudden-transition view of development. Such an implicit commitment may derive some of its power from the "representational warehouse" model of cognition and development that rose to prominence in the cognitive revolution. An alternative model of cognition and development, grounded in dispositional patterns of responding to stimuli, more naturally accommodates dissociative phenomena in development and highlights mechanisms for self-regulation and for fashioning and deploying representations, or depictions, in a uniquely human way.
\end{abstract}

\section{Prevalence of Implicit Antigradualism}

When asked explicitly, most researchers readily grant that cognitive development can be gradual, fractured and inconstant, riddled with dissociations and regressions, unstable and context-dependent through long transitional periods, and (shall we say) multifariously messy. Views like Siegler's (1996), Schwitzgebel's (1999), and Woolley's (2006), which stress this complexity in development, generally meet with broad agreement in principle (if not always in detail). On the face of it, the overall picture is uncontentious, with the only question being where, and to what degree, we find dissociative phenomena.

In our view, this apparent consensus is an illusion - and indeed is itself a dissociative phenomenon. What researchers say in the abstract tends to diverge from how they behave in designing and interpreting individual experiments. Consider, for example, the common practice of trying to pin down the particular points in development at which major cognitive transitions occur rather suddenly. Gradualism and dissociation, of course, speak against such sudden shifts.

Another telltale sign of an implicitly antigradualist model is the tendency to favor one particular type of experiment as the best measure of the presence or absence of a particular skill and to devalue all other experimental approaches to that skill-especially those that suggest an earlier or later age of acquisition-as misleading, artifactual, laden with extraneous demands, subject to resolution by simpler

As both authors contributed equally to this commentary, the order of authorship is alphabetical.

Correspondence concerning this article should be addressed to Eric Schwitzgebel, Department of Philosophy, University of California, Riverside, CA 92521-0201. Electronic mail may be sent to eschwitz@ucr.edu. strategies, or as really testing some other (perhaps related) skill or understanding. If one is thoroughly committed to the idea of development as gradual and fractured, one should be completely unsurprised, unpuzzled, and comfortable with broad dissociations: One should expect that the best portrayal of any flexible and interesting cognitive achievement will involve a wide variety of equally valid tests of exactly the same skill or understanding, passed at substantially different ages. To expect otherwise is to reject the gradualist, dissociationist view. So also is gradualism implicitly rejected by those who insist on the real and full presence of the relevant knowledge as soon as the child displays it by any legitimate, nonartifactual measure, and by those who insist on breaking the skill in question into component parts, each acquired at a distinct age. Sometimes, of course, it makes perfectly good sense to break a skill into component parts or to dismiss certain experiments as artifactual or laden with extraneous demands; but to take such possibilities, implicitly or explicitly, as the natural default options, to feel unsettled unless one can resolve experimental diversity by such maneuvers, to assume that the question "does the child know X?" always admits of a yes-or-no answer once the experiments are properly understood, is to fail to take gradualism to heart.

What could account for this persistent dissociation between research theory and practice? One answer immediately suggests itself: Pragmatically, it is far easier to deal with decisive tests and clean answers. Both our science and our cognition handle simple quanta much better than tangled masses. We seek out crisp lines, simplifying and idealizing the data, explaining away or ignoring what does not neatly fit.

(C) 2006 by the Society for Research in Child Development, Inc. All rights reserved. 0009-3920/2006/7706-0004 
But the lure of crisp lines is not enough, we suspect, to account for these proclivities. A deeper explanation may be found in the cognitive revolution itself. For while that revolution has led to a great era of research, it has also encouraged a dominating model of the mind that is structurally incompatible with a thorough-going gradualism. We do not say this model should be altogether abandoned. But there may be other models of mind equally congenial to the cognitivist spirit that do a better job capitalizing on the dissociative phenomena so prevalent in development. In these comments, we gesture toward one such model (or class of models); discuss why exploiting this model opens up useful new ways of thinking about data that already exist; and point toward future directions for research made pressing by this model but that are not as easily visible from the more traditional cognitivist perspective.

\section{Two Models}

Consider two ways of describing an understanding of false belief:

The Representational Warehouse Model: Somewhere in the mind is a box containing all the propositions the child takes to be true that are required for attributing false beliefs. Such propositions include: other people have minds; among the things the mind contains are states whose job it is to represent how things are in the world; people acquire such states through their interactions with the world; sometimes these states are out of date because the world changes without people having access to those changes; one way not to have access to a change is not to see it occur; etc. From this storehouse of propositions, the child may infer what a given person will believe under a variety of circumstances, responding to various prompts - for example, "where will Sally look for her marble?" - in various appropriate verbal and nonverbal ways. Learning about mental states in general and beliefs in particular is essentially a matter of adding propositional items to the storehouse.

The Dispositional Model: Learning about mental states in general and beliefs in particular is learning patterns of response to a variety of scenarios such as: "Let's give Mommy a present for her birthday. Do you know what her favorite color is? ... That's not Mommy's favorite color, that's your favorite color! What's Mommy's favorite color?" Or, "Look, Tommy's having trouble finding his crayons. He thinks they're in the cupboard. But you know where they really are. Can you help Tommy find his crayons?" Or, "Let's surprise Daddy. He won't think we're hiding in the closet. Let's keep very still and shout 'surprise' when he opens the door!' With sufficient training, the child comes ever more swiftly and consistently to respond to scenarios of this sort in an adultlike way, with fewer external supports and crutches, even coming to initiate her own plans along similar lines. To come to understand the nature of mental states is, in essence, nothing more than coming to be inclined, or "disposed," to generate the right sorts of verbal and nonverbal actions and responses (including patterns of thought) in a diversity of environments, regardless of how that pattern is actually achieved.

One approach might underwrite the other, incorporating elements of the other in a subordinate or derivative way, but not in the most fundamental architecture. Maybe the stored representations are causally responsible for the dispositional patterns, the patterns of response to stimuli. Or conversely, maybe the dispositional patterns arise from diverse causal structures that are not themselves well characterized in terms of high-level representations, yet support high-level representational attributions much as pixels on a screen support the attribution of an image, however the pixels come to be so arranged.

We hope you will agree that something in the representational warehouse model resonates with mainstream cognitive science, with the model of mind bequeathed us by the cognitive revolution, with representationalism in psychology and philosophy; and something in the dispositional model resonates with behaviorism, with connectionism and dynamic systems theory, with contextualist and Vygotskian approaches to development, with dispositional and "interpretationist" approaches in philosophy. We do not pretend, of course, that these simplified descriptions capture all the subtlety and nuance in such views fully and properly developed or that this dichotomy exhausts the alternatives. Theoreticians also sometimes blend the two approaches (e.g., in dual process theories where one process may be viewed as more warehouse-like, the other more purely dispositional). But here is our thought: The two models so described are primed to handle dissociations and gradualism differently. In particular, fundamental to the warehouse view is the antigradualist assumption that any particular representation at any particular time either is or is not stored in the warehouse.

\section{What Are the Empirical Risks?}

While these models present rather different starting points for thinking about cognition and development, we do not put them forward as exclusive 
possibilities, with one or the other of them capturing what is literally true about how the mind works. Our attitude is pragmatic. We ask: Which model, which approach, which habit of thinking gives researchers the more lucid and fruitful science? Is development more helpfully and productively thought of as resembling the acquisition and deployment of stored representations or as a gradual shaping of patterns of responsiveness? Both approaches are compatible with much of the existing research. Both approaches can make sense of, and see value in, descriptions of the child's developmental trajectory. Yet the expectations and questions the two models invite are very different.

A dispositional view, for instance, positively predicts dissociation, gradual change, and contextual contingency. On this view, inconsistency is the normal starting point. Since dispositions are shaped in different contexts, it takes a certain amount of work to get one's myriad verbal and nonverbal responses all in line-work that involves, for instance, self-regulation, attention, practice, repetition. It takes work to become robustly successful in making allowances for false belief in circumstances of deception as well as inadvertency, in games and in earnest, in parents and in peers; it takes work not to be taken in, or at least influenced, by wishes and superstitions; it takes work to resist the impulse to treat the longer thing as bigger in situations of conservation. In fact, many of us never reach perfect ease and fluency in such skills, even if we do a lot better than 4-year-olds (more on this below).

The representational warehouse view may of course accommodate our imperfect mastery of false belief, or any other dissociation - but only by positing some sort of additional structure or functional impediment to proper deployment of the representation. If there is a representation in the warehouse with the content "beliefs can be false" or "you cannot make more liquid by pouring it from one container to another," then there is some explaining or excusing to do when the competencies those representations are supposed to underwrite are not consistently displayed. Maybe the system is distracted or overloaded or misled and the relevant representation is not retrieved. Maybe the representation is encoded in a format or functional location that some systems have access to but not others. Maybe multiple conflicting representations are encoded - in single or in distinct representational systems - with different conditions of deployment. Each of these types of explanation involves empirical commitments of various sorts and opens up new directions for inquiry.

The representational warehouse model thus seems to have the more cumbersome explanatory structure for dealing with dissociation. But this is not necessarily a vice. Because it must invoke mechanisms and structures to explain dissociation, it suggests various testable hypotheses. Sophisticated representational warehouse models may even predict dissociations in advance and be supported by them. If the commitments and suggestions of such models pan out, if they lead to further fruitful studies (of the mechanisms of representational retrieval, say, or a taxonomy of functionally discrete systems), then we have been well served by the models. But conversely - and this is the risk essential to science - if things do not pan out so well, if such mechanisms seem only excuses, ad hoc manipulations of self-preservation in the face of troublesome data, without testable empirical content or helpful direction to future research, then we should look for another approach.

In contrast, the dispositional approach, abstractly considered, may seem too accommodating to the data. After all, dispositions can seemingly change in any number of ways - inconstantly, probabilistically, clustering or clumping in one pattern or another. While this may make the view hard to falsify, it leaves us at a loss regarding what to predict. To have scientifically exciting content, the dispositional model must be made specific and detailed enough to anticipate particular patterns of change or dissociation - as perhaps in Woolley's idea that dissociations relate to differences in goal, or in the Vygotskian idea that they turn on the variable effects of social "scaffolding," or in the efforts by Thelen and others (e.g., Thelen, Schöner, Scheier, \& Smith, 2001) to build testable mathematical models of patterns of behavior. One of the key projects for advocates of dispositional and dissociative approaches to development should be to continue to articulate and clarify such ideas, so as to generate substantive, risky commitments of the sort that can direct research.

\section{Mechanisms of Dispositional Regulation}

Another, somewhat different way to address this issue of empirical risk is to note how the dispositional view has complementary explanatory demands to the representational warehouse view. As noted above, the representational warehouse view seems to require extra structure to explain dissociative phenomena. In the representational warehouse, constancy is the suggested norm; inconstancy requires explanation, some way of accommodating the gap between competence and performance. On the dispositional model, however, inconstancy is the suggested norm, and extra structure is required to explain constancy - to explain, that is, the seamless nature of mature competency, the apparent 
uniformity of knowledge in both verbal and nonverbal modalities across a range of disparate contexts. If advocates of dispositional models can propose specific mechanisms geared toward producing and maintaining such constancy-mechanisms that are unnecessary or of merely secondary importance on a representational warehouse approach - then substantive empirical commitments will follow, commitments that are themselves risky and directive, as well as sufficiently different from those of the representational warehouse view to allow a comparison of the two approaches.

So what sorts of mechanisms might be required to produce and maintain dispositional consistency? Some might be external, consisting of various types of environmental feedback and support. Consider, for instance, Subbotsky's explanation of why children often manifest more mature knowledge in their verbal responses to questions about magical phenomena than their behavioral responses. Quoting Woolley: "He suggests that children are aware of the scientific worldview possessed by most adults, and recognize that, when discussing such matters, this worldview is valued over a magical one. Yet when left to their own devices, children feel freed of these expectations and behave in accord with a more magical view of the world" (p. 1546, our emphasis). This quote makes clear both the effectiveness and the limitations of external feedback and support, and it generates further testable predictions about when children will and will not behave in ways suggestive of magical thinking.

To ensure stable, consistent patterns of response, children must also make use of expectations and norms in a self-regulatory way. That is to say, they must begin to take over much of the regulatory work that others do to get and keep their dispositional tendencies in line (McGeer, 2001; McGeer \& Pettit, 2002). Perhaps young children's tendency to decide on "favorite colors," "lucky numbers," and such, in part serves as a kind of practice at keeping their dispositional preferences consistent and predictable. Similarly, techniques such as sitting on one's hands, repeating rules out loud, and practicing developing skills in low-demand contexts may serve in part to self-regulate and control otherwise unruly dispositions. Furthermore, if such a view is correct, a plethora of less directly observable and subpersonal mechanisms will surely also play a crucial role in self-regulation - such as in preventing and correcting false starts in speech or in reaching behavior. A particularly interesting type of self-regulation combines the environmental with the purely self-regulatory - as when an adult publicly announces a plan to lose weight or quit smoking in part to create social pressure to conform to that very plan, or when one deliberately (or at least not accidentally) chooses environments that tend to call forth behavior in accord with one's general dispositions and espoused values.

We suggest that if such external and self-regulatory mechanisms can be found, and if they play a crucial role in preserving dispositional consistency, that hints of something right in the dispositional view; and to the extent they are unnecessary or secondary, that speaks against the view. We think some current findings in the developmental literature, as well as in the adult cognitive and social psychological literature, can be interpreted accordingly. For example, numerous social psychological studies attest to the variability of our apparent beliefs, values, and traits across environments, and suggest that being consistent and predictable requires a certain amount of both internal and external pressure and regulation. Anecdotal evidence for this idea also abounds. Consider, for instance, the self-regulatory work it takes (e.g., self-admonition, reminders) to avoid falling victim to the gambler's fallacy, to adhere to one's decided strategy in the stock market, to overcome the automatic deployment of racial stereotypes, to counteract superstitious or magical thinking, or to properly trust instruments rather than one's proprioceptively guided judgments (about up and down and so forth) when flying an airplane.

One particularly interesting phenomenon, relevant to our much-vaunted theory-of-mind capacities, comes from Keysar, Lin, and Barr (2003). They have recently shown a dissociation between our reflective grip on the difference between our own and others' beliefs - as, for instance, in the standard false belief task - and our capacity to act in ways that show a similar grasp of the situation. In their paradigm, participants were put in a situation where they knew some information that another person (the "director") did not know-for instance, they knew the contents of a closed bag, visible only from their perspective. All normal adults would of course be perfectly capable of saying how their knowledge differs from the director's. But in the Keysar et al. study, participants were instead asked to do something at the director's behest, where the director's request could be interpreted according to the director's knowledge or according to the participant's knowledge. For example, the director might say "move the tape," while a cassette tape is in plain view and a roll of Scotch tape is hidden in a closed bag known only to the participant. Most participants showed at least some tendency to act based on an egocentric misinterpretation of the director's re- 
quest - thus acting more like 3-year-olds in the standard false belief task. Of course, adults who initiated the wrong act were often able to self-correct (i.e., self-regulate) according to their more sophisticated reflective understanding - although there was also a tendency to rely on corrective feedback from the environment in the person of the director, suggesting that adults continue to use others in their regulatory regimes. This fits neatly with a dispositional model of the sort we advocate: Our dispositional tendencies tend to remain unruly unless kept in line by the self- (and other-) regulatory practices at which adults become rather adept, at least in many circumstances.

In defense of the representational warehouse model, one could always say that different subsystems employ or have access to different conflicting representations, or that in some situations representations are retrieved while in others they are not. But our bet is that in many such cases the phenomena will be inconstant, more gradual, more probabilistic, less divisible into discrete subsystems with discretely present or absent representational contents, than is easily harmonized with this view. Ironically, the misplaced demand for sharp edges may make these phenomena appear more chaotic than they would on a more gradualist, self-regulatory approach.

\section{Rethinking the Role of Representations}

In reviewing the evidence for verbal-behavioral dissociations in various domains, Woolley notes that sometimes more mature patterns of response are shown in verbal than in behavioral measures and sometimes it is the other way around. This prompts her to ask (p. 1542) whether it is really plausible to think there are "specific roles" played by these modalities. While we agree entirely with her main conclusion - that researchers should not treat either modality as a decisively better measure of the child's "true" cognitive condition-we do think the verbal modality often plays at least one interestingly specific role, a role closely tied to the child's developing capacity for self-regulation.

Consider the difference between learning to catch a fly ball the usual way, by trial and error with balls arriving at various trajectories and speeds, and learning to catch a fly ball by deliberately following this rule: Fixate your gaze on the ball, start running, and adjust your speed so that the angle of gaze remains constant. Sometimes it may be useful to consider such a rule in learning how to catch; and yet at the same time the rule seems at best a representational crutch - something eventually to be set aside in favor of more fluid competency as the perceptual-motor system gets trained up. Or consider the difference between navigating campus by means of an external (or imagined) map as opposed to following habitual routes through well-known areas. Using a map might sometimes lead to more efficient campuscrossing than adapting and stringing together habits, especially if one needs to cross in an unusual direction; in other conditions, using a map might be less efficient, or unnecessary, or misleading.

But don't we always use rules, of some sort or other, in catching a fly ball (even if we are unaware of those rules)? Don't we always use map-like representations to get across campus (even if we have no literal visual image of a map)? Cognitive science has reaped many rewards from characterizing the mind in this way; and we are happy to endorse such characterizations insofar as they make room for various competing models of what it means to have or use "representations," including models (like the dispositional view) that take a nondiscrete, vague, and embodied approach to representation (see $\mathrm{p}$. 1558). But there is also a more constrained, and here atypical, use of the term "representation" we find attractive. It tracks a distinction between, on the one hand, merely behaving in a way that seems to embody some rule or piece of knowledge and, on the other, engaging in a special and uniquely human kind of knowing activity: that is, making an explicit representation of things - depicting them - in words, pictures, or symbols. This is the distinction between simply knowing how to get somewhere and telling a friend how to get there; between walking delicately across thin ice and saying that the ice is thin (or drawing a cautionary picture of a man falling in). It would seem most apt to call this specialized skill for making representations the skill of representing; but given how this term is standardly used in cognitive science, it may be better to call it the skill of depicting how things are.

Treating depiction as a distinctive skill (a skill that, we are sure, develops dissociatively, in fits and starts) highlights certain patterns in the dissociative phenomena of development: We should expect dissociations between the child's ability to show knowledge through depiction and her ability to show knowledge through direct, nondepictional intervention in the world. Woolley brings this out to some extent in her discussion of goals. She points out that in some experiments, the child may understand her task as one of "conveying knowledge" - in our preferred terms, the child aims to say or otherwise depict how the world is. In other tasks, the child might understand her goal to be what we might call 
"interventional" - to sort cards, for example, or to help someone find something, or to engage in exploratory investigations. Given the great difference between depictional and interventional skills, it should be utterly unsurprising that children sometimes learn how to depict a situation before they can intervene effectively, and sometimes the reverse. For instance, in the card-sorting task, the child learns to sort cards one way, and then acquires new depictional knowledge of a rule for sorting them a new way (by being told the rule). Naturally, such a child might be able to say what the new rule is before she can enact it reliably. By contrast, a child may have extensive experience intervening in particular types of situations that involve ignorance or false belief-for instance, in helping someone find something, in playing hide-and-seek, in surprising and tricking people - but struggles to deliver appropriate answers when asked to depict another's state of knowledge, even in such familiar situations. This may account for some of the dissociations we see in various theory-of-mind tasks.

We cannot defend such a take on the theory-ofmind literature in any detail here, obviously; our point is only to emphasize that having a clear understanding of depiction or representation as a specialized skill in its own right can give us a methodologically rigorous way to analyze existing data on dissociative phenomena and can open new avenues of research - analyses and avenues it may be easy to miss on a model of the mind according to which "representation" is something we all do constantly, by the very fact of cognizing.

A second reason to highlight depiction as a specialized skill connects with the idea of self-regulation. For once children begin to master the capacity of depicting-mainly saying or thinking-how things are or ought to be, they can use this capacity to consciously direct and regulate their own verbal and nonverbal behavior. We saw examples of this in the previous section and also above in the use of maps and in learning to catch. Thus, although we resist the idea that (depictional) verbal measures provide a special and privileged indicator of the child's "true" state of knowledge, we do think the capacity plays a special role in the child's pulling her dispositional tendencies into the alignment characteristic of mature competency. We would like to see more empirical investigation of this regulative process, including for instance: what the early signs of self-regulation are; how children increasingly come to use their depictional capacities to articulate and regulate what they are or should be doing; and the relationship between subpersonal, habitual, and deliberate conscious monitoring of aberrant thought and action.

We conclude by mentioning a third, cautionary reason for emphasizing the highly distinctive and specialized nature of our human skill of depicting (or representing). Verbal and symbolic depictions are a powerful technology for conveying and manipulating information. As with many such technologies, including most notably clockwork mechanisms (in earlier days) and digital computers (more recently), it is inviting to theorize about the mind in these terms, to portray mental processes as analogous to or even identical with such familiar tools. It may thus seem natural or even inevitable to suppose that depiction, or representation, is what our minds are doing behind our backs, as it were-and not just what our minds are doing behind our backs but also what the minds of nondepicting creatures are doing behind their backs. It is critical to see this metaphorical extension of our depicting capacity for what it is - a metaphorical extension that gives researchers one optional model for explaining how cognitive systems generally work. Indeed, despite the model's natural allure, we think the very existence of widespread dissociative phenomena is beginning to show the limitations of a simple representationalism that supposes the discrete presence or absence of stored, "warehoused," representational depictions. Our hope is that something like the dispositional model is on the right track, and that whatever it lacks in intuitive appeal it can soon make up for in empirical adequacy and theoretical suggestiveness.

\section{References}

Keysar, B., Lin, S., \& Barr, D. J. (2003). Limits on theory of mind use in adults. Cognition, 89, 25-41.

McGeer, V. (2001). Psycho-practice, psycho-theory and the contrastive case of autism. Journal of Consciousness Studies, 8, 109-132.

McGeer, V., \& Pettit, P. (2002). The self-regulating mind. Language and Communication, 22 (5-7), 281-299.

Schwitzgebel, E. (1999). Gradual belief change in children. Human Development, 42, 283-296.

Siegler, R. S. (1996). Emerging minds. Oxford, UK: Oxford University Press.

Thelen, E., Schöner, G., Scheier, C., \& Smith, L. B. (2001). The dynamics of embodiment: A field theory of infant perseverative reaching. Behavioral and Brain Sciences, 24, $1-86$.

Woolley, J. D. (2006). Verbal-behavioral dissociations in development. Child Development, 77, 1539-1553. 